$$
\begin{gathered}
\xi_{2}= \pm \frac{1}{2}\left\{-2 \frac{\left(\frac{\partial g}{\partial \xi}\right)_{0}}{g_{0}}\right\} h^{2}+\epsilon, \\
\xi_{1}= \pm \frac{1}{2}\left\{-\frac{1}{3 g_{0}}\left[\left(\frac{\partial g}{\partial \xi}\right)_{0}+4 \omega^{2} \sin \phi_{0} \cos \phi_{0}\right]\right\} h^{2}+\epsilon^{\prime},
\end{gathered}
$$

where $\epsilon$ and $\epsilon^{\prime}$ are infinitesimals of order higher than that of $h^{2}$. In order to remove the ambiguity in sign let us observe that since the latitute $\phi$ lies between $-90^{\circ}$ and $+90^{\circ}$, it follows that $\cos \phi>0$, and therefore, by relation $(40),(\partial j / \partial s) / j$ and $d^{2} y / d x^{2}$ are either both positive or both negative. Hence it follows from (45) and Theorem 8 that for the curve $d$, - $(\partial g / \partial \xi)_{0} / g_{0}$ and $\left(d^{2} z / d \tau^{2}\right)_{0}$ are either both positive or both negative; and from (50) and Theorem 9 that for the curve $c^{\prime}$, $-1 / g_{0}\left\{(\partial g / \partial \xi)_{0}+2 \omega^{2}\right.$ sin $\left.2 \phi_{0}\right\}$ and $\left(d^{2} z / d \tau^{2}\right)_{0}$ are either both positive or both negative. Furthermore, we assumed $\xi_{1}$ and $\xi_{2}$ to be positive toward the equator. Consequently if for definiteness we suppose (as shown in Fig. 5) that for curve $d,\left(d^{2} z / d \tau^{2}\right)_{0}>0$ and for curve $c^{\prime},\left(d^{2} z / d \tau^{2}\right)_{0}<0$, it follows that $\xi_{2}=-P_{1} T<0$ and $\xi_{1}=T C^{\prime}>0$. Therefore in the above expressions for $\xi_{2}$ and $\xi_{1}$ the lower signs must be used and thus we have

$$
\begin{gathered}
\xi_{2}=\frac{\left(\frac{\partial g}{\partial \xi}\right)_{0}}{g_{0}} h^{2}, \\
\xi_{1}=\left\{2 \omega^{2} \sin 2 \phi_{0}+\left(\frac{\partial g}{\partial \xi}\right)_{0}\right\} \frac{h^{2}}{6 g_{0}},
\end{gathered}
$$

to terms of order not higher than the second in $h$, whence

$$
\text { S.D. }=\xi_{1}-\xi_{2}=\left\{2 \omega^{2} \sin 2 \phi_{0}-5\left(\frac{\partial g}{\partial \xi}\right)_{0}\right\} \frac{h^{2}}{6 g_{0}},
$$

which is formula (I).

\title{
NOTE ON SOLVABLE QUINTICS.
}

$$
\text { BY PROFESSOR F. N. COLE. }
$$

(Read before the American Mathematical Society, January 2, 1915.)

THE substance of the following paper was included several years ago in my university lecture course on the theory of 
groups. Being laid away for seasoning and final touches, it was destroyed in the fire of last October, along with the rather lengthy computations under Lagrange's theorem involved in the transition from the equations of Runge to those here employed. I have succeeded in restoring the paper from the lecture notes of my students, and am moved to publish it now in order that whatever value it may have may not be further exposed to destruction. The process of solution seems to be reduced to the simplest terms, and I have not been able to find that it has been anticipated.

The quintic is supposed to have been reduced (by Tschirnhausen transformation) to the form $x^{5}+\alpha x+\beta=0$. In his paper in the Acta Mathematica, volume 7 (1885), Runge obtained the sextic resolvent

$$
(v-\alpha)^{4}\left(v^{2}-6 \alpha v+25 \alpha^{2}\right)=5^{5} \beta^{4} v,
$$

where $v=\left(x_{1} x_{2}+x_{2} x_{3}+x_{3} x_{4}+x_{4} x_{5}+x_{5} x_{1}\right)^{2}$, the $x$ 's being the roots of the quintic, and expressed the coefficients of solvable (irreducible) quintics in terms of two parameters. The condition for solvability is of course that the resolvent sextic shall have a rational root. The function $v$ belongs to the group of order 20 , and the problem is substantially to calculate by radicals the four Lagrange functions

$$
\begin{array}{ll}
\tau_{1}=x_{1}+\epsilon x_{2}+\epsilon^{2} x_{3}+\epsilon^{3} x_{4}+\epsilon^{4} x_{5}, & \tau_{3}=x_{1}+\epsilon^{3} x_{2}+\epsilon x_{3}+\epsilon^{4} x_{4}+\epsilon^{2} x_{5}, \\
\tau_{2}=x_{1}+\epsilon^{2} x_{2}+\epsilon^{4} x_{3}+\epsilon x_{4}+\epsilon^{3} x_{5}, & \tau_{4}=x_{1}+\epsilon^{4} x_{2}+\epsilon^{3} x_{3}+\epsilon^{2} x_{4}+\epsilon x_{5} .
\end{array}
$$

These last belong to the identical group, their fifth powers to a group of order 5, the combinations $\sigma_{1}=\tau_{1}^{5}+\tau_{4}^{5}, \sigma_{2}=\tau_{2}{ }^{5}$ $+\tau_{3}^{5}$ to a group of order 10 , and finally $5^{3} \rho=\sigma_{1}+\sigma_{2}$ to the same group of order 20 as Runge's $v$.

I find now the following series of resolvent equations:

$$
\begin{gathered}
4^{5} \alpha^{5}\left(\rho^{2}+6 \beta \rho+25 \beta^{2}\right)=(\rho+25 \beta)(\rho+5 \beta)^{5} ; \\
\sigma_{1}+\sigma_{2}=5^{3} \rho, \quad \sigma_{1} \sigma_{2}=-\frac{5^{6}}{2^{4}}\left(\rho^{2}+30 \beta \rho+125 \beta^{2}\right) ; \\
\tau_{1}^{5}+\tau_{4}^{5}=\sigma_{1}, \quad \tau_{1}^{5} \tau_{4}^{5}=\frac{5^{6}}{2^{6}} \frac{(\rho+25 \beta)^{3}}{\sigma_{2}-\sigma_{1}}=-\tau_{2}^{5} \tau_{3}^{5} .
\end{gathered}
$$

As an example, the equation $x^{5}+15 x+12=0$ gives $\rho=60, \quad \sigma_{1}+\sigma_{2}=5^{4} \cdot 12, \quad \sigma_{1} \sigma_{2}=-5^{8} \cdot 108, \quad \sigma_{1}=-5^{4} \cdot 6$, 
$\sigma_{2}=5^{4} \cdot 18 . \quad \tau_{1}^{5}+\tau_{4}^{5}=-5^{4} \cdot 6, \quad \tau_{1}^{5} \tau_{4}^{5}=3^{5} \cdot 5^{5}, \quad \tau_{1}^{5}=$ $-1875+525 \sqrt{10}, \quad \tau_{4}^{5}=-1875-525 \sqrt{10} . \quad \tau_{2}^{5}+\tau_{3}^{5}$ $=5^{4} \cdot 18, \quad \tau_{2}^{5} \tau_{3}^{5}=-3^{5} \cdot 5^{5}, \quad \tau_{2}^{5}=5625+1800 \sqrt{10}, \quad \tau_{3}^{5}$ $=5625-1800 \sqrt{10} . \quad 5 x=\tau_{1}+\tau_{2}+\tau_{3}+\tau_{4}$.

The connection with Runge's resolvent is effected by the relation

$$
\rho=-5 \beta \frac{v-5 \alpha}{v-\alpha},
$$

by which equation (1) may be verified. The relation

$$
\sigma=\frac{-5^{4} \beta}{2(v-\alpha)}\left(v-5 \alpha+\sqrt{v^{2}-6 \alpha v+25 \alpha^{2}}\right),
$$

which includes the preceding and gives the key to equations (2) and (3), was worked out by Lagrange's theorem.

Columbia University, April 6, 1915.

\section{THE MADISON COLLOQUIUM LECTURES ON MATHEMATICS.}

Part I: On Invariants and the Theory of Numbers. By LEONARD Eugene Dickson. New York, American Mathematical Society, 1914.

THE number of new mathematical systems which may be characterized as distinct mutations, whose discovery or development is to be credited to American research, has shown a marked increase within a few decades. The reviewer of Professor Dickson's Lectures of the Madison Colloquium volume has the satisfaction of recording one of these great discoveries, his theory of classes in invariant theory, and of observing how as a result of this discovery, number theory, which long had little contact with the theory of invariants, now has very much in common with it. Dickson's technical memoirs in which the theory of classes and the invariant theory of modular forms were first expounded appeared in 1909 . And while the material and indeed much of the method also of the Colloquium Lectures are new, they are dominated by the theory of classes and may, therefore, be regarded as a superstructure of the system founded in his 1909 papers. Lecture I may be regarded also, as introductory to the theory as a whole. 